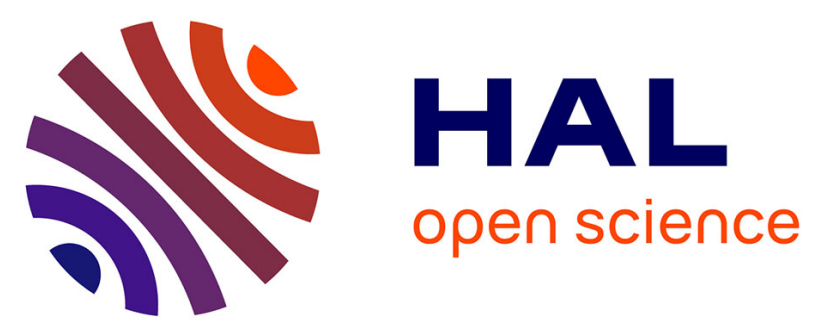

\title{
The use of higher proportions of platelet-rich plasma to enrich microfat has negative effects :A Preclinical Study
}

Maxime Abellan Lopez, Baptiste Bertrand, F. Kober, Mohamed Boucekine,

Marc de Fromont de Bouailles, Marie Vogtensperger, Monique Bernard, Dominique Casanova, Jéremy Magalon, Florence Sabatier

\section{To cite this version:}

Maxime Abellan Lopez, Baptiste Bertrand, F. Kober, Mohamed Boucekine, Marc de Fromont de Bouailles, et al.. The use of higher proportions of platelet-rich plasma to enrich microfat has negative effects:A Preclinical Study. Plastic and Reconstructive Surgery, 2020, 145 (1), pp.130-140. 10.1097/PRS.0000000000006406 . hal-02325382v1

\section{HAL Id: hal-02325382 \\ https://hal.science/hal-02325382v1}

Submitted on 15 Feb 2022 (v1), last revised 16 Feb 2022 (v2)

HAL is a multi-disciplinary open access archive for the deposit and dissemination of scientific research documents, whether they are published or not. The documents may come from teaching and research institutions in France or abroad, or from public or private research centers.
L'archive ouverte pluridisciplinaire HAL, est destinée au dépôt et à la diffusion de documents scientifiques de niveau recherche, publiés ou non, émanant des établissements d'enseignement et de recherche français ou étrangers, des laboratoires publics ou privés. 


\section{The Use of Higher Proportions of Platelet-Rich Plasma to Enrich Microfat Has Negative Effects: A Preclinical Study}

Maxime Abellan Lopez, M.D., M.Sc., Baptiste Bertrand, M.D., Ph.D., Frank Kober, Ph.D., Mohamed Boucekine, Ph.D., Marc De Fromont De Bouailles, M.D., Marie Vogtensperger, Monique Bernard, Ph.D., Dominique Casanova, M.D., Jeremy Magalon, Pharm.D., Ph.D., Florence Sabatier, Pharm.D., Ph.D.

From the Plastic Surgery Department and the Cell Therapy Department, Hôpital de la Conception, AP-HM; Public Health, Chronic Diseases and Quality of Life, Research Unit, INSERM, INRA, C2VN, Aix-Marseille Univ, CNRS, CRMBM Pathologie; Aix Marseille University; and the Cell Therapy Department, Hôpital Hautepierre. France.

Received for publication June 3, 2018; accepted February 7, 2019. The first two authors contributed equally to the study.

Disclosure: The authors report no conflicts of interest. All aspects of this study were funded by the "Fondation des gueules cassées" in 2015. DOI: 10.1097/PRS.0000000000006406

\section{Abstract}

Background: Platelet-rich plasma improves engraftment after fat transfer. However, the effects of platelet dose have never been investigated. The authors used magnetic resonance imaging to compare surviving graft volumes in mice after administration of four different formulations (microfat alone, and three platelet-rich plasma-enriched microfat mixes).

Methods: The authors used a random, double-blinded, fat transfer protocol using three different platelet levels: 1 million (low-dose), 500 million (mediumdose), and 1000 million (high-dose) platelets $/ \mathrm{ml}$, and fat alone (control). The authors grafted $0.4 \mathrm{ml}$ of the $70 / 30$ platelet-rich plasma-enriched microfat mixtures $(0.4$ million, 200 million, and 400 million platelets per $0.12 \mathrm{ml}$ for the low-dose, medium-dose, and high-dose mixtures, respectively) or $0.4 \mathrm{ml}$ of microfat alone into 22 nude mice and monitored surviving graft volumes every month for 3 months. Then, the authors histologically analyzed all grafts to assess neoangiogenesis status and fat integrity.

Results: Three-dimensional magnetic resonance imaging showed that the median surviving graft volumes at 3 months were 9.5 percent (interquartile range, 0 to 25 percent; $p=0.003$ ) (high-dose), 4.1 percent (interquartile range, 0 to 18 percent; $p=0.001$ ) (medium-dose), and 18 percent (interquartile range, 8 to 38 percent; $p=0.41$ ) (low-dose) compared to 36 percent (interquartile range, 28 to 53 percent) for the control value. The histologic integrity of microfatalone grafts was significantly better than those of the other grafts, although the high-dose and low-dose grafts exhibited higher levels of neoangiogenesis.

Conclusion: Higher platelet levels in microfat grafts were associated with poor graft survival in nude mice; a clinical review would be appropriate.

(Plast. Reconstr. Surg. 145: 00, 2020.) 


\section{BACKGROUND}

The positive preclinical results of autologous cell-based therapy have opened a new research approach toward regenerative surgery; certain products may exhibit useful clinical applications. Platelet-rich plasma, an autologous blood product, is now readily available, and its use has become the standard of care for several abnormalities. $(1,2)$ Platelet-rich plasma can be prepared in the operating room using any of several commercial kits. (3) Growth factors synthesized by platelets in platelet-rich plasma [including vascular endothelial growth factor (VEGF), platelet-derived growth factor (PDGF), and transforming growth factor- $\beta$ (TGF- $\beta$ )] may explain the observed regenerative capacity. (4) However, platelet levels differ markedly among platelet-rich plasma produced manually or using kits. (5) As described previously by Nguyen et al., autologous microfat transfer is simply obtained through dedicated fat harvesting that uses a multiperforated cannula with holes of $1 \mathrm{~mm}$ diameter allowing harvest of smaller lobules of fat (approximately $600 \mu \mathrm{m}),(6)$ and particularly useful when restoring soft facial tissue. $(7,8)$ The aim is to restore or increase the volume of subcutaneous fat, and improve skin elasticity and thickness. Recently, plastic surgeons have added plateletrich plasma to injected fat, (9-11) hypothesizing that growth factors produced in vivo would enhance the neovascularization, proliferation, and differentiation of adipose stem cells, improving patient outcomes. However, no consensus on the appropriate fat-to-platelet-rich plasma ratio has emerged, and the plateletrich plasma used has varied. Thus, the effects of platelet-rich plasma on long-term fat volume remain unclear. The suggestion that platelet-rich plasma composition (in terms of platelet numbers) may affect the final graft volume has not been explored.

Here, we explored the long-term viabilities of various platelet-rich plasma/microfat mixes in an immunosuppressed murine model of subcutaneous, human microfat transfer. We blindly (and randomly) evaluated three 30/70 percent platelet-rich plasma/microfat mixes containing 1 million (low-dose), 500 million (medium-dose), and 1000 (high-dose) million platelets $/ \mathrm{ml}$, compared to a microfat-alone group (control group). We used magnetic resonance imaging to blindly monitor graft survival every month for 3 months and also performed semiquantitative and qualitative histologic analyses of the 3-month grafts.

\section{MATERIALS AND METHODS}

\section{Cell Therapy Products}

Human blood and adipose tissue were harvested from two healthy volunteers (42- and 47-year-old women who were not taking any drugs) during aesthetic liposuction; both gave written informed consent. Microfatalone and plateletrich plasma/microfat mixes were prepared in a class A microbiological safety cabinet located in the culture and cell therapy department of our university hospital.

\section{Platelet-Rich Plasma Preparation}

Platelet-rich plasma was manufactured using a previously described method. (12) Briefly, whole blood was collected into $8.5-\mathrm{ml}$ neutral tubes each containing $1.5 \mathrm{ml}$ of adenosine citrate dextroseacid solution (catalogue no. BDB8651; Fenwal Inc., Portland, Ore.) followed by centrifugation at $130 \mathrm{~g}$ for 15 minutes (Multifuge Heraus 3 S-R centrifuge; Thermo-Scientific, Indianapolis, Ind.); the supernatant served as the platelet-rich plasma. After platelet counting, various volumes of platelet-rich plasma were added to three 11$\mathrm{ml}$ conical tubes (NUNC, catalogue no. 56423; Thermo-Scientific) followed by centrifugation at $250 \mathrm{~g}$ for 10 minutes to pellet the platelets, which then were resuspended in platelet-poor plasma and homogenized. Onemilliliter syringes were used to draw up $0.3-\mathrm{ml}$ amounts of suspension containing 1 million, 500 million, or 1000 million platelets.

\section{Platelet-Rich Plasma Characterization}

Levels of platelets, leukocytes, and red blood cells in whole blood and platelet-rich plasma were measured by means of fluorescent flow cytometry (XN-10; Sysmex Corp., Kobe, Japan). The levels of 11 cytokines and growth factors with inflammatory actions [interleukin (IL)-1 $\beta$, tumor necrosis factor- $\alpha$, IL-6, interferon (IFN)- $\gamma$ ], antiinflammatory actions (IL-10, IL-1RA), or regenerative properties (PDGF-AB and PDGF-BB, VEGF, nerve growth factor, TGF- $\beta 1$, basic fibroblast growth factor) in activated platelet-rich plasma (12) were simultaneously measured using a Magpix instrument (Luminex xMAP Technology; Luminex, Inc., Austin, Texas).

\section{Microfat Harvesting and Preparation}

Microfat was collected using a previously described technique (6) from patients undergoing abdominal, internal knee, and peritrochanteric liposuction under general anesthesia; the microfat was considered surgical residue. Briefly, liposuction was performed using a st'Rim cannula (st'Rim Pack; Thiebaud Biomedical Devices, Margencel, France) with a 2-mm-diameter hole and a blunt tip less than $1 \mathrm{~mm}$ in diameter, drawing on the plunger of a 10-cc Luer-Lok (BD Medical, Franklin Lakes, N.J.) syringe to exert a 2cc negative pressure space. The $10-\mathrm{ml}$ syringe and the cannula were connected in a closed system to a sterile collection bag through a Fat Lock System (Benew Medical, Melesse, France). The oily and hematic 
phases were separated by means of microcentrifugation (Universal $320 \mathrm{R}$ centrifuge; Hettich, Tuttlingen, Germany) at $1200 \mathrm{~g}$ for 3 minutes, and the hematic phase was discarded (i.e., the Coleman method (13) ). Then, 1 -ml syringes were partially filled with $0.7-\mathrm{ml}$ amounts of the microfat suspension.

\section{Mice}

The study was approved by our National Animal Care and Ethics Committee (no. 2016010615549974). We required a minimum of 16 mice to reveal a significant difference (15 percent) in terms of platelet-rich plasma/microfat (compared to microfat alone) graft survival at an alpha risk of 5 percent and a beta risk of 80 percent, given that we randomized the injection sites. Twenty-two immunosuppressed female BALB/ cAnNRj-Foxnu1 nu (9) mice (8 weeks of age and weighing $20 \mathrm{~g}$ ) were purchased from Janvier Laboratories (Genest-Saint-Isle, France) and allowed to acclimatize for 2 weeks before the experiments.

\section{Recipient Sites}

We used four subcutaneous injection sites (the left and right shoulders and hips) to engraft the three microfat/platelet-rich plasma mixes and microfat alone. The four preparations evaluated were randomly injected among these sites. Randomization was performed in blocks of four to avoid any effects of injection site on graft survival. The 22 mice were divided randomly into two groups (Fig. 1).

\section{Injection}

Mice were injected under general anesthesia established be means of halogenated gas inhalation (Sevoflurane; Baxter, Maurepas, France) within 2 hours following harvesting human blood and microfat. Platelet-rich plasma and microfat were mixed at 30/70 percent proportions. For the three conditions using platelet-rich plasma, two 1-ml syringes were connected and mixed by gentle back-and-forth shaking of the syringes five times to finally obtain a volume of $1 \mathrm{ml}$ of a homogeneous mixed product. [See Video 1 (online), which shows platelet-rich plasma and microfat being mixed at 30/70 percent proportions. For the three conditions using platelet-rich plasma, two 1-ml syringes were connected and mixed by gentle back-and-forth shaking of the syringes five times to finally obtain a volume of $1 \mathrm{ml}$ of a homogeneous mixed product.] We injected 0.4-ml amounts of platelet-rich plasma/microfat mixes or microfat alone in a random and blinded manner into each of the four subcutaneous injection sites per mouse, under sterile conditions, using the 21gauge, blunt reinjection cannula of the st'Rim pack. Thus, the platelet doses (per $0.12 \mathrm{ml}$ ) were 0.4 million, 200 million, and 400 million, respectively. [See Video 2 (online), which shows subcutaneous injection into nude mice.]

\section{Magnetic Resonance Imaging}

We used magnetic resonance imaging to measure graft volumes at all four sites on day 1 and at 1,2 , and 3 months after injection in mice under general anesthesia induced by halogenated gas inhalation (see above) and subjected to cardiorespiratory monitoring. We used a 4.7-T, smallanimal Biospec 47/30 scanner (Bruker Biospin, Ettlingen, Germany). We used a 6-cm transmit volume resonator antenna, and a 3-cm-diameter decoupled receive surface antenna (Rapid Biomedical, Rimpar, Germany). The acquisition protocol (Bruker Paravision Version 4.0) featured three perpendicular slices (the fast low angle shot sequence), localization of a coronal section (by means of fast low angle shot), acquisition of 25 axial multisectional images (rapid acquisition with relaxation enhancement; effective echo time, $30 \mathrm{msec}$; repetition time, 5 seconds), and acquisition of an additional 25 axial multisectional images (rapid acquisition with relaxation enhancement; effective echo time, $60 \mathrm{msec}$; repetition time, 5 seconds). Volumetric analyses were performed in a blinded manner on an OsiriX model 9.5 DICOM viewer (Pixmeo, Bernex, Switzerland) (Fig. 2). [See Video 3 (online), which shows posttreatment estimation of surviving graft volumes using an OsiriX 9.5 Digital Imaging and Communications in Medicine viewer (Pixmeo, Bernex, Switzerland).]

\section{Histologic Analyses}

After 3 months, the mice were killed by means of cervical dislocation under general anesthesia. We created quadrangular skin incisions and removed en bloc the skin, the panniculus carnosus, and each microfat graft. After paraffin infusion, all specimens were stained with conventional hematoxylin-phloxine-eosin and examined under a light microscope (Axiophot; Zeiss, Oberkochen, Germany). A blinded independent pathologist used a semiquantitative scale ( 0 , absent; 1 , minimally present; 2 , minimally to moderately present; 3 , moderately present; 4 , moderately to extensively present; and 5 , extensively present) $(14,15)$ to evaluate fat cell integrity; neoangiogenesis and the status of cysts; vacuolation, fibrosis, necrosis, and inflammation, using NDP2 viewing software (Hamamatsu Photonics, Hamamatsu, Japan).

\section{Statistical Analyses}

The magnetic resonance imaging volumes of resorption are expressed as median [interquartile range (first quartile to third quartile)]. The semiquantitative histologic data are expressed as mean \pm standard error. The Nonparametric Analysis of Longitudinal Data in Factorial Experiments (nparLD package) from R Version 3.5.2 software (R Foundation for Statistical Computing, Vienna, Austria) was performed for magnetic 
resonance imaging volume analysis. The Wald-type test statistic was used to compare magnetic resonance imaging-derived graft volumes over time compared to those 1 day after injection (baseline volumes). The interaction term between sites and time was tested in this statistical model. We completed this global analysis by comparing the graft survival of the three platelet-rich plasma-enriched microfat mixes versus graft survival of the microfat alone at each time of magnetic resonance imaging evaluation with a KruskalWallis nonparametric test (SPSS Version 17.0; SPSS, Inc., Chicago, III.). We performed the Bonferroni test and we presented the Bonferroni adjusted $p$ values for the pairwise comparisons. Semiquantitative histologic data were analyzed by means of analysis of variance and the Tukey post hoc test (SPSS Version 17.0). Values of $p<0.05$ were considered to be significantly different.
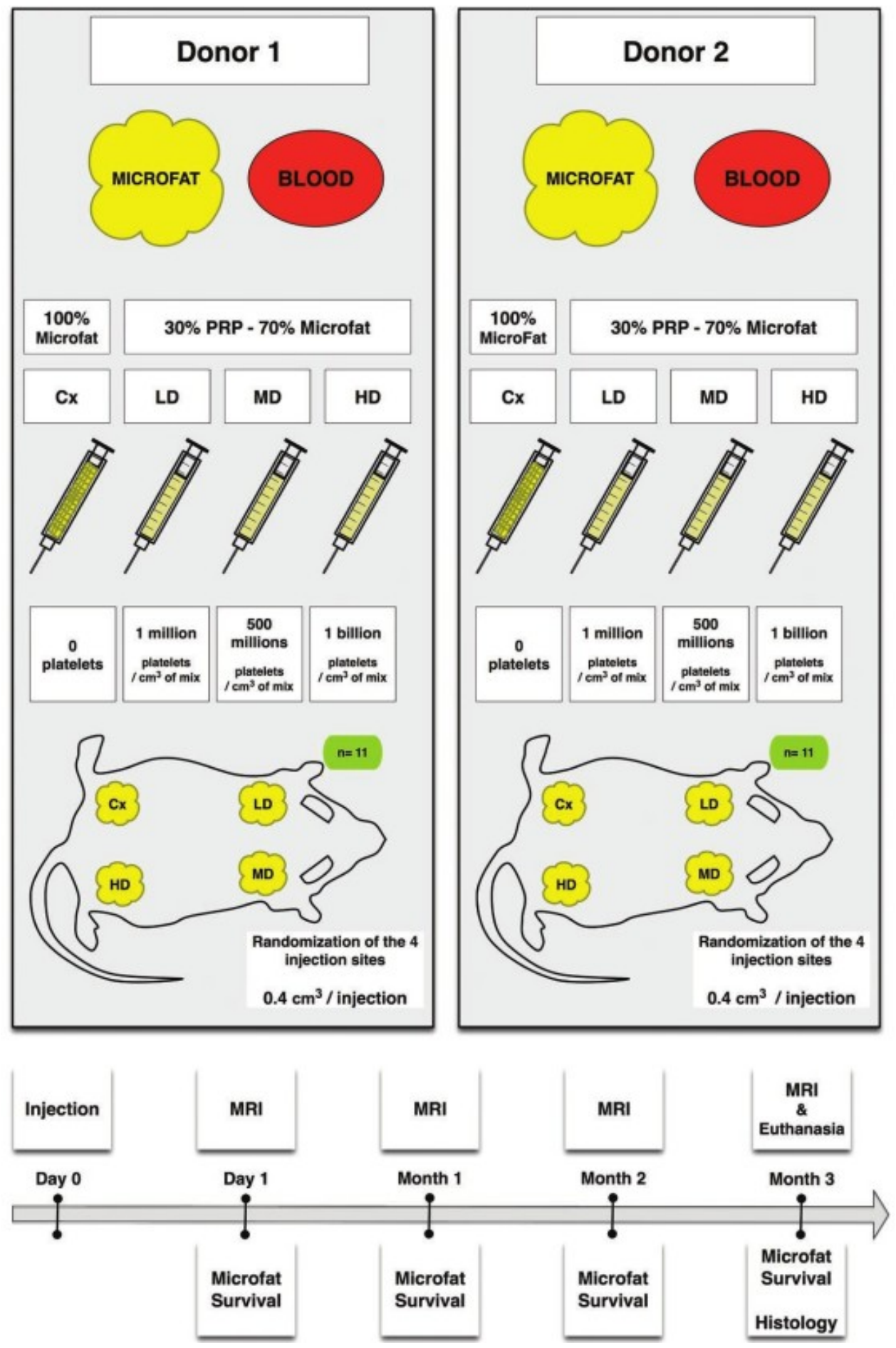

Fig. 1. Design of the study and the three platelet-rich plasma/microfat mixes. PRP, platelet-rich plasma; MRI, magnetic resonance imaging; $C x$, control; $L D$, low-dose; $M D$, medium-dose; HD, high-dose. 

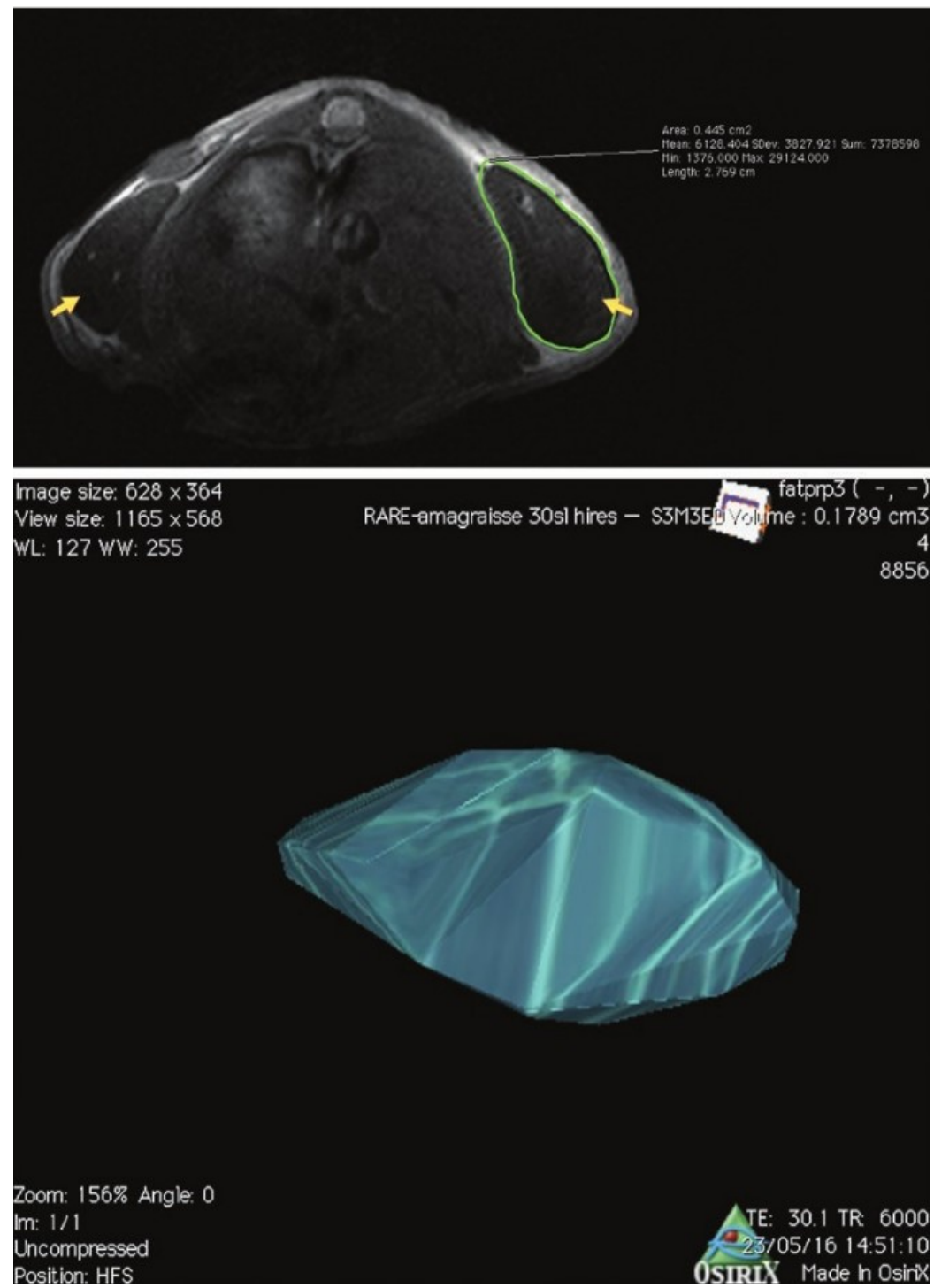

Fig. 2. Magnetic resonance imaging analyses of graft survival. (Above) Graft survival (yellow arrows) in the mouse back; two-dimensional analyses. (Below) Three-dimensional reconstruction to allow analyses of graft survival.

\section{RESULTS}

\section{Cell Therapy Products}

We collected $80 \mathrm{ml}$ of microfat from either patient, and obtained $40-\mathrm{ml}$ amounts of purified microfat. The platelet-rich plasma manufactured from patients 1 and 2 contained 94.9 percent and 95.2 percent platelets, respectively, with a few red blood cells and leukocytes. The platelet-rich plasma from patient 1 did not release any inflammatory cytokines (IL-1 $\beta$, IL-6, or IFN- $\gamma$ ), whereas that of patient 2 did; the platelet-rich plasma of patient 2 also produced more antiinflammatory cytokines and regenerative growth factors than the platelet-rich plasma of patient 1, particularly in terms of IL-10 (16fold difference) and VEGF (10-fold difference) (Table 1). 


\section{Magnetic Resonance Imaging}

The median fat graft volumes 1 day after injection were $0.34 \mathrm{ml}$ (interquartile range, 0.29 to $0.38 \mathrm{ml}$ ), Relative composition of PRP, \% $0.24 \mathrm{ml}$ (interquartile range, 0.21 to Platelets $0.27 \mathrm{ml}$ ), $0.25 \mathrm{ml}$ (interquartile range, 0.21 to $0.31 \mathrm{ml}$ ), and $0.25 \mathrm{ml}$ (interquartile range, 0.21 to $0.28 \mathrm{ml}$ ) for the control, low-dose, medium-dose, and high-dose groups, respectively $(0.4 \mathrm{ml}$ was initially injected). The difference between the control (85 percent of volume remaining) and the mixes was significant $(p=0.001)$. The volume of fat graft resorption was significantly higher over time for the high-dose group $(p<0.001)$, for the medium-dose group $(p<0.001)$, and for the lowdose group ( $p=0.02)$ compared with the microfat-alone group. This volume did not differ for the three platelet-rich plasma-enriched microfat groups ( $p=$ not significant).

Leukocytes

Red blood cells

PRP releasate $(\mathrm{pg} / \mathrm{ml}) *$

Inflammatory cytokines

$\begin{array}{lcc}\text { IL-1 } \beta & 3.5 & 57 \\ \text { TNF- } \alpha & \text { ND } & 330 \\ \text { IL-6 } & \text { ND } & 110 \\ \text { IFN- } \gamma & \text { ND } & 950 \\ \text { Antiinflammatory cytokines } & & \\ \text { IL-10 } & 23 & 350 \\ \text { IL-1RA } & 400 & 2900 \\ \text { egenerative growth factors } & & \\ \text { PDGF-BB } & 0.1 & 0.1 \\ \text { VEGF } & 310 & 3300 \\ \text { NGF } & 52 & 430 \\ \text { TGF- } \beta & 0.4 & 0.2 \\ \text { Basic FGF } & \text { ND } & \text { ND }\end{array}$

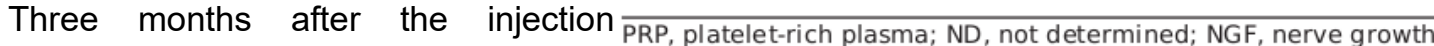
procedure, the residual median factor; FGF, fibroblast growth factor.

volumes (referenced to the day-1 *Per 1 million platelets per cubic centimeter of mix.

volumes) were 36 percent (interquartile Table 1: Biological Characterization of Platelet-Rich Plasma Manufactured range, 28 to 53 percent), 18 percent (interquartile range, 8 to 38 percent),

Using Materials from Patients 1 and 2 4.1 percent (interquartile range, 0 to 18 percent), 9.5 percent (interquartile range, 0 to 25 percent) for the control, low-dose, medium-dose, and high-dose groups, respectively (Fig. 3).

\section{Months Graft Survival Curve}

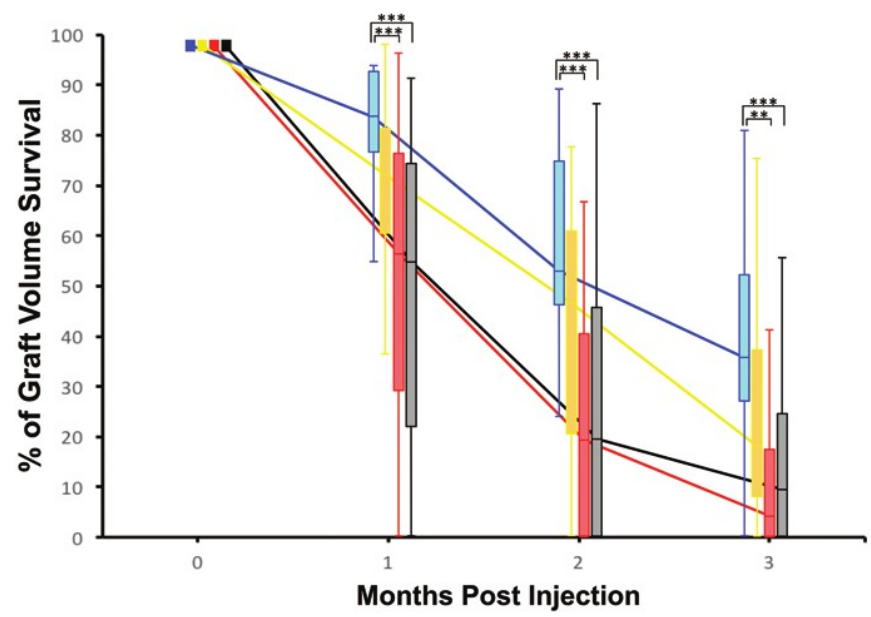

Thus, the median percentage of volume losses at 3 months was 64 percent (interquartile range, 47 to 72 percent) in the control group compared to 82 percent (interquartile range, 62 to 92 percent) ( $p=$ $-\square-\mathrm{cx}$ 0.41), 96 percent (interquartile range, 82 to 100 percent) ( $p=0.001$ ), and 91 percent (interquartile range, 75 to 100 percent $)(p=0.003)$ in the low-dose, medium-dose, and high-dose groups, respectively. The losses did not differ between grafts prepared using plateletrich plasma of patients 1 and $2(p=0.24)$. The injection site did not interact with time in the analysis of resorption ( $p=0.06)$.

Fig. 3. Three-month median graft survival curves: microfat alone (Cx); microfat/platelet-rich plasma low-dose (LD), medium-dose (MD), and high-dose (HD). Significant differences were evident at months 1,2 , and $3{ }^{* *} p<0.01 ;{ }^{* * *} p$ $<0.001)$. 
After the animals were killed at 3 months after injection, macroscopic differences in the residual grafts of the

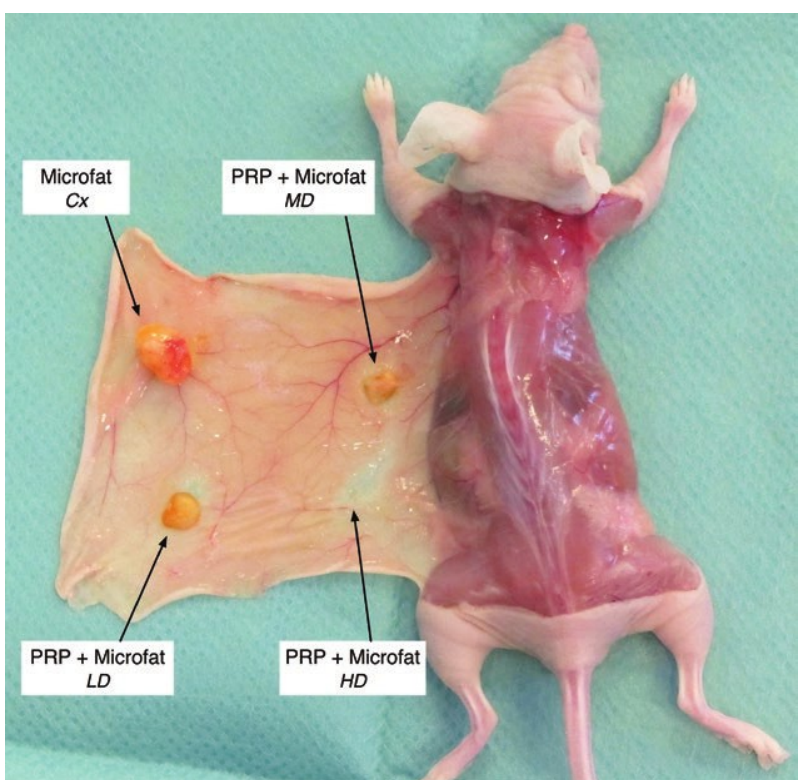

Fig. 4. The results of dissection 3 months after microfat injection. Cx, control; PRP, platelet-rich plasma; LD, low-dose platelets; $M D$, medium-dose platelets; $H D$, high-dose platelets. control and platelet-rich plasma/micro-fat mix groups were apparent. Microfat grafts lying between the panniculus carnosus and adjacent muscles were supplied by vessels of the former tissue (Fig. 4).

The adipose tissue had been remodeled and different cells were organized in concentric layers, with viable adipocytes and neovessels on the periphery. In addition, there was a deeper inflammatory front evidencing significant fibrosis and pseudocysts. Finally, the graft center had necrotic microfat lacking nucleated adipocytes or any other viable cells or neovessels (Fig. 5). The semiquantitative histologic data are summarized in Figure 6 . The integrity of the control grafts was significantly better compared with the microfat/platelet-rich plasma grafts $(p<0.0001)$. In terms of neovascularization, the control and lowdose mix grafts scored significantly higher than the mediumdose mix graft $(p<0.05)$, but lower than the high-dose mix graft $(p<0.001)$. In terms of cyst/vacuole status, control grafts scored significantly better (fewer cysts/vacuoles) than mixed grafts $(p=0.04)$. The extent of fibrosis was similar in all grafts. In terms of necrosis, the control and low-dose mix scores were significantly lower than those of the other graft mixes $(p<0.001)$. In terms of inflammation, the high-dose mix grafts scored significantly higher than the other grafts $(p=0.048)$.

\section{DISCUSSION}

The potential of fat grafting has attracted increasing interest in volume resaturation. However, long-term fat graft retention varies greatly, and the addition of platelet-rich plasma may enhance graft survival by releasing proangiogenic factors that enhance vascularization. Plastic surgeons commonly inject platelet-rich plasma, although the data in the literature are contradictory and graft preparation and injection methods vary greatly. We are the first to use magnetic resonance imaging to measure the graft survival of four platelet-rich plasma/microfat formulations, including three with different levels of platelets. Platelet-rich plasma and microfat were mixed at 30/70 percent proportions because an earlier rheologic study showed that such mixtures remained soft. (16) In addition, it is useful to be precise about the need for platelet-rich plasma considering that an approximately $30-\mathrm{ml}$ mix (thus including $10 \mathrm{ml}$ of platelet-rich plasma) is usually required to restore human soft facial tissue. Platelet-rich plasma platelet levels range from 0.2 to 10.8 billion given available data concerning platelet-rich plasma preparation devices. (3) Thus, $30 \mathrm{ml}$ of platelet-rich plasma/microfat mix may contain from 6 to 400 million platelets $/ \mathrm{ml}$. We thought it useful to test an extremely high platelet dose, although such doses are not clinically available. Unexpectedly, no platelet-rich plasma/microfat mix afforded better engraftment than microfat alone. Indeed, the medium-dose and highdose mixes were associated with graft volume loss (compared with the control) at 3 months after transfer; the surviving volumes of the control and low-dose grafts were similar. The magnetic resonance imaging data were in agreement with the histologic data, revealing better integration of mature adipocytes of microfat alone compared with those of the three microfat/platelet-rich plasma mixes. Use of the medium-dose and high-dose mixes was associated with inflammation, necrosis, and vacuolation, but the mixes did not reduce extracellular matrix synthesis or increase fibrosis. Surprisingly, the high-dose mix exhibited significantly more neovascularization than all other formulations, despite the poor graft survival. Our results contradict those of most prior studies that have assessed the impact of plateletrich plasma on graft survival $(15,17-23)$; most have reported positive effects of platelet-rich plasma. However, graft survival in those studies was not assessed by means of magnetic resonance imaging; more approximate (and less expensive) methods were used (a liquid overflow method, ultrasound, and histomorphometric analyses). The only study reporting nonsignificant difference between platelet-rich plasma-enriched fat and fat grafts alone is that of Por et al., (15) who used a nonactivated platelet-rich plasma preparation containing 56 million platelets $/ \mathrm{ml}$. 

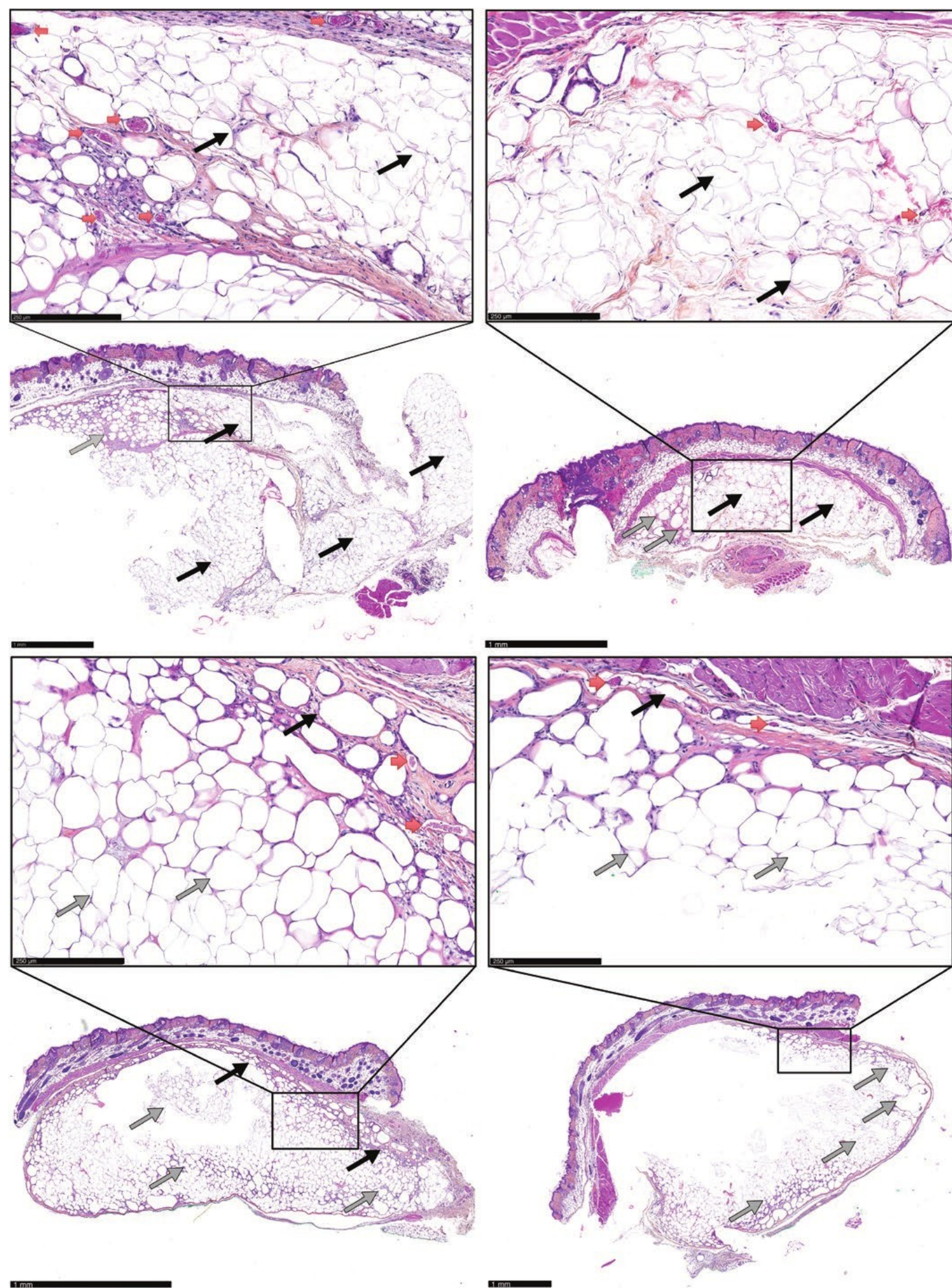

Fig. 5. Hematoxylin-phloxine-eosin staining of skin and grafted adipose tissue 3 months after microfat injection. (Above, left) Fat-alone group. Many neovessels (red arrows) and viable clusters of nucleated adipocytes (black arrows) can be seen, although a focus of necrotic collagenous tissue is apparent (gray arrows). (Above, right) Platelet-rich plasma/microfat in the low-dose group. Rare neovessels (red arrows) viable adipocytes with nuclei (black arrows) exhibiting only slight changes (pseudocysts and coagulation, albeit only focally) (gray arrows) can be seen. (Below, left) Platelet-rich plasma/microfat medium-dose group. Peripheral neovessels (red arrows) and nonviable adipocyte ghosts lacking visible nuclei (black arrows) and exhibiting numerous vacuolar changes and periphera pseudocysts (gray arrows) can be seen. (Below, right) Platelet-rich plasma/microfat in the high-dose group. Multiple peripheral neovessels (red arrows); large areas exhibiting necrotic vacuolar changes, nonviable adipocyte ghosts, and many peripheral pseudocysts (gray arrows) can be seen. 

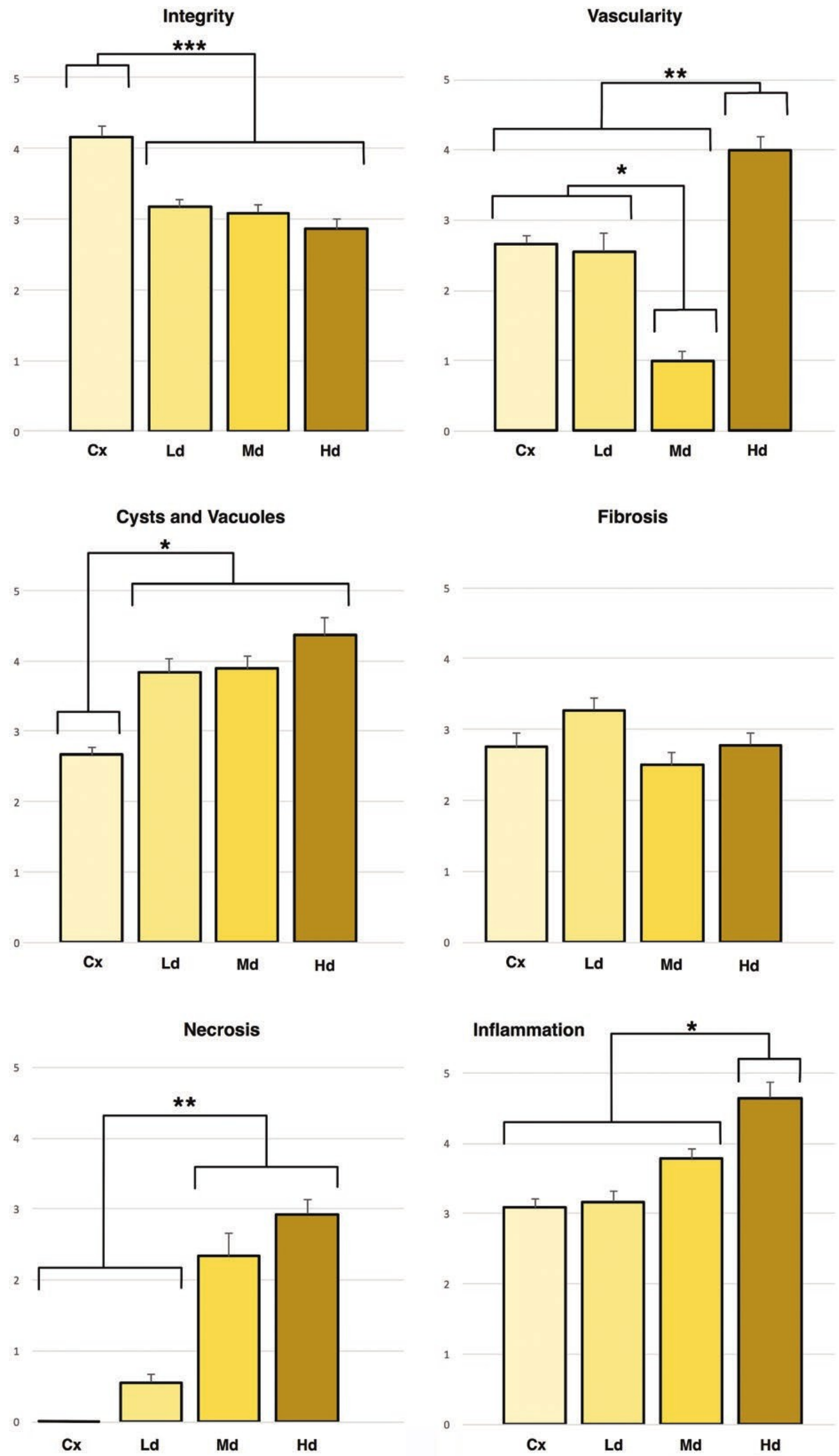

Fig. 6. Semiquantitative histologic analyses of grafts formed from microfat alone (Cx) and low-dose (Ld), medium-dose (Md), and highdose $(\mathrm{Hd})$ platelet-rich plasma/microfat $\left({ }^{*} p<0.05 ;{ }^{* *} p<0.001 ;{ }^{* * *} p<0.0001\right)$. (Above, left) Microfat integrity; (above, right) vascularity of the microfat graft; (center, left) cyst/vacuole status; (center, right) fibrosis status; (below, left) necrosis status; and (below, right) tissue inflammation status. 
Table 2. Review of Experimental Studies Comparing the Graft Survivals of Platelet-Rich Plasma/Fat Mixtures and Fat Alone

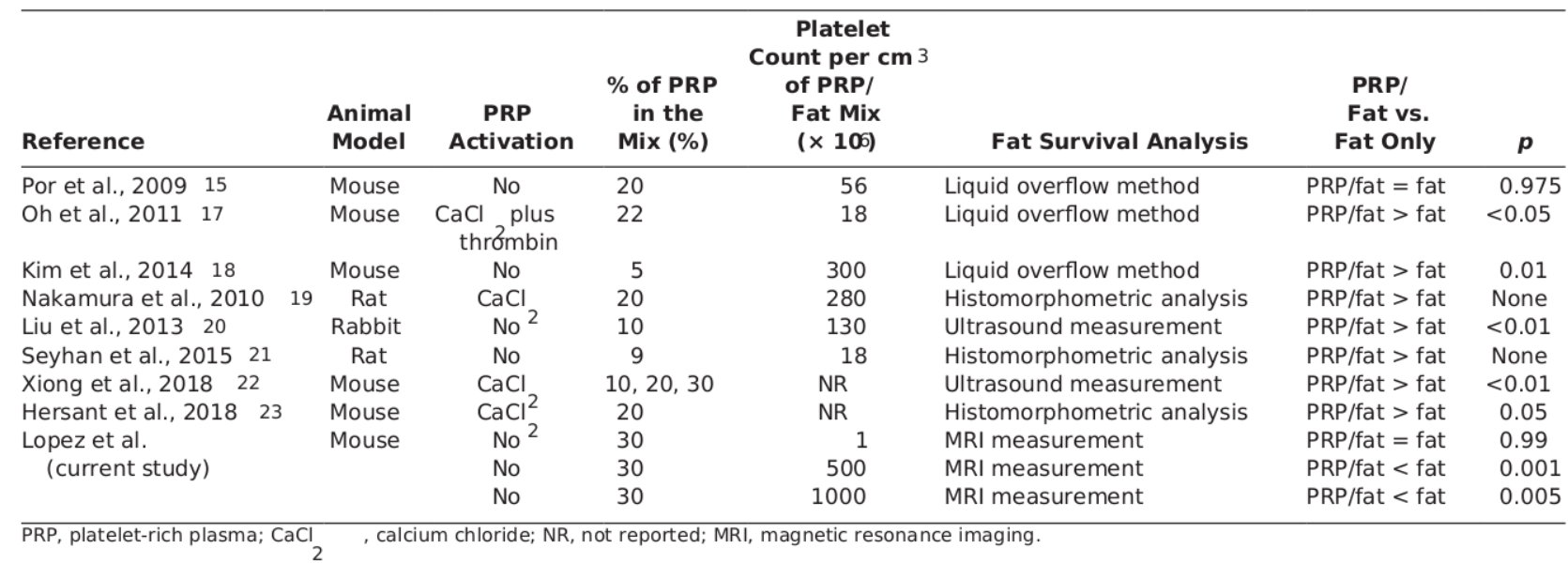

Table 2 summarizes the mix characteristics and outcomes of other preclinical studies, and highlights the variability in platelet-rich plasma preparation and the fact that the platelet-rich plasma percentage and platelet numbers per milliliter of mix are key when comparing studies. From a clinical viewpoint, our results are consistent with the absence of any benefit of platelet-rich plasma enrichment recently reported by Willemsen et al. 24 in a double-blinded, placebo-controlled, randomized trial that compared the use of fat alone versus a platelet-rich plasma/fat mix for facial rejuvenation. In that study, platelet-rich plasma did not improve outcomes in terms of skin elasticity or graft volume maintenance. Unfortunately, the report does not detail either the volume or biological characteristics of the injected platelet-rich plasma, but it is suggested that high platelet levels may be counterproductive, possibly because they trigger undesirable cellular differentiation or adipose stem cell differentiation toward a fibroblast-like phenotype. recently reported Indeed, Chignon-Sicard et al.25 that TGF- $\beta$ released by platelet-rich plasma may have both antiadipogenic and promyofibroblastic effects on adipose stem cells. In a randomized controlled trial, excess TGF- $\beta$ released by plateletrich plasma used to treat knee osteoarthritis correlated with a reduced functional score. 26 TGF- $\beta$ neutralization enhances muscle regeneration and prevents fibrosis. 27 We found that the TGF- $\beta$ levels were similar in the platelet-rich plasmas of both patients but of course increased 500- and 1000fold when the medium-dose and high-dose mixes were used (compared to the low-dose mix). 105618 Fat Survival Analysis PRP/ Fat vs. Fat Only Finally, one of our most interesting findings was that 40 percent of the graft volume was lost 1 day after injection of each of the platelet-rich plasma/microfat mixes compared to 15 percent of graft volume lost 1 day after injection of microfat alone, suggesting that the platelet-rich plasma immediately spread subcutaneously. Thus, the injection volume should be increased to reflect early plateletrich plasma loss. The major limitation of our study is that we used an immunodeficient mouse model (human fat recipients will not be immunodeficient). In addition, it is important to state that different fat preparations (i.e., decantation or filtration) or platelet-rich plasma activation could lead to different results. Finally, the number of volunteers used $(n=2)$ for microfat and platelet-rich plasma preparations limits any investigation in the potential influence of growth factor and cytokines profile on microfat retention. CONCLUSIONS Inclusion of platelet-rich plasma during microfat grafting did not enhance graft survival; indeed, high-dose platelets compromised survival. Magnetic resonance imaging revealed immediate graft volume loss (including the totality of the injected platelet-rich plasma volume) 1 day after injection. Further magnetic resonance imaging- based studies are needed.

Baptiste Bertrand, M.D. Department of Plastic and Hand Surgery La Conception Hospital 147 Boulevard Baille 13005 Marseille, France

baptiste.bertrand@gmail.com

\section{REFERENCES}

1. Paterson KL, Hunter DJ, Metcalf BR, et al. Efficacy of intraarticular injections of platelet-rich plasma as a symptomand disease-modifying treatment for knee osteoarthritis: The RESTORE trial protocol. BMC Musculoskelet Disord. 2018;19:272.

2. Sclafani AP, Azzi J. Platelet preparations for use in facial rejuvenation and wound healing: A critical review of current literature. Aesthetic Plast Surg. 2015;39:495-505.

3. Magalon J, Chateau AL, Bertrand B, et al. DEPA classification: A proposal for standardising PRP use and a retrospective application of available devices. BMJ Open Sport Exerc Med. 2016;2:e000060. 
4. Marx RE, Carlson ER, Eichstaedt RM, Schimmele SR, Strauss JE, Georgeff KR. Platelet-rich plasma: Growth factor enhancement for bone grafts. Oral Surg Oral Med Oral Pathol Oral Radiol Endod. 1998;85:638-646.

5. Magalon J, Bausset O, Serratrice N, et al. Characterization and comparison of 5 platelet-rich plasma preparations in a single-donor model. Arthroscopy 2014;30:629-638.

6. Sautereau N, Daumas A, Truillet R, et al. Efficacy of autologous microfat graft on facial handicap in systemic sclerosis patients. Plast Reconstr Surg Glob Open 2016;4:e660.

7. Nguyen PS, Desouches C, Gay AM, Hautier A, Magalon G. Development of micro-injection as an innovative autologous fat graft technique: The use of adipose tissue as dermal filler. J Plast Reconstr Aesthet Surg. 2012;65:16921699.

8. Alharbi Z, Opländer C, Almakadi S, Fritz A, Vogt M, Pallua N. Conventional vs. micro-fat harvesting: How fat harvesting technique affects tissue-engineering approaches using adipose tissue-derived stem/stromal cells. J Plast Reconstr Aesthet Surg. 2013;66:1271-1278.

9. Luck J, Smith OJ, Mosahebi A. A systematic review of autologous platelet-rich plasma and fat graft preparation methods. Plast Reconstr Surg Glob Open 2017;5:e1596.

10. Serra-Mestre JM, Serra-Renom JM, Martinez L, Almadori A, D'Andrea F. Platelet-rich plasma mixed-fat grafting: A reasonable prosurvival strategy for fat grafts? Aesthetic Plast Surg. 2014;38:1041-1049.

11. Jin R, Zhang L, Zhang YG. Does platelet-rich plasma enhance the survival of grafted fat? An update review. Int $\mathrm{J}$ Clin Exp Med. 2013;6:252-258.

12. Bausset O, Giraudo L, Veran J, et al. Formulation and storage of platelet-rich plasma homemade product. Biores Open Access 2012;1:115-123.

13. Coleman SR. Structural fat grafting: More than a permanent filler. Plast Reconstr Surg. 2006;118(Suppl):108S-120S.

14. Ramon Y, Shoshani O, Peled IJ, et al. Enhancing the take of injected adipose tissue by a simple method for concentrating fat cells. Plast Reconstr Surg. 2005;115:197-201; discussion 202-203.

15. Por YC, Yeow VK, Louri N, Lim TK, Kee I, Song IC. Plateletrich plasma has no effect on increasing free fat graft survival in the nude mouse. J Plast Reconstr Aesthet Surg. 2009;62:1030-1034.

16. Ghazouane R, Bertrand B, Philandrianos C, et al. What about the rheological properties of PRP/microfat mixtures in fat grafting procedure? Aesthetic Plast Surg. 2017;41:1217-1221.

17. Oh DS, Cheon YW, Jeon YR, Lew DH. Activated platelet-rich plasma improves fat graft survival in nude mice: A pilot study. Dermatol Surg. 2011;37:619-625.

18. Kim DY, Ji YH, Kim DW, Dhong ES, Yoon ES. Effects of platelet-rich plasma, adipose-derived stem cells, and stromal vascular fraction on the survival of human transplanted adipose tissue. J Korean Med Sci. 2014;29(Suppl 3):S193-S200.

19. Nakamura S, Ishihara M, Takikawa M, et al. Platelet-rich plasma (PRP) promotes survival of fat-grafts in rats. Ann Plast Surg. 2010;65:101-106.

20. Liu B, Tan XY, Liu YP, et al. The adjuvant use of stromal vascular fraction and platelet-rich fibrin for autologous adipose tissue transplantation. Tissue Eng Part C Methods 2013;19:1-14.

21. Seyhan N, Alhan D, Ural AU, Gunal A, Avunduk MC, Savaci N. The effect of combined use of platelet-rich plasma and adipose-derived stem cells on fat graft survival. Ann Plast Surg. 2015;74:615-620.

22. Xiong BJ, Tan QW, Chen YJ, et al. The effects of platelet-rich plasma and adipose-derived stem cells on neovascularization and fat graft survival. Aesthetic Plast Surg. 2018;42:1-8.

23. Hersant B, Bouhassira J, SidAhmed-Mezi M, et al. Should platelet-rich plasma be activated in fat grafts? An animal study. J Plast Reconstr Aesthet Surg. 2018;71:681-690.

24. Willemsen JCN, Van Dongen J, Spiekman M, et al. The addition of platelet-rich plasma to facial lipofilling: A doubleblind, placebo-controlled, randomized trial. Plast Reconstr Surg. 2018;141:331-343.

25. Chignon-Sicard B, Kouidhi M, Yao X, et al. Platelet-rich plasma respectively reduces and promotes adipogenic and myofibroblastic differentiation of human adipose-derived stromal cells via the TGF $\beta$ signalling pathway. Sci Rep. 2017;7:2954.

26. Louis ML, Magalon J, Jouve E, et al. Growth factors levels determine efficacy of platelets rich plasma injection in knee osteoarthritis: A randomized double blind noninferiority trial compared with viscosupplementation. Arthroscopy 2018;34:1530-1540.e2.

27. Li H, Hicks JJ, Wang L, et al. Customized platelet-rich plasma with transforming growth factor $\beta 1$ neutralization antibody to reduce fibrosis in skeletal muscle. Biomaterials 2016;87:147-156. 